\title{
Ist die Zukunft der Mobilität elektrisch?
}

\section{Fachtagung der Österreichischen Gesellschaft für Energietechnik im OVE}

\author{
J. Vavra
}

Online publiziert am 20. Februar 2017

(C) Springer Verlag Wien 2017

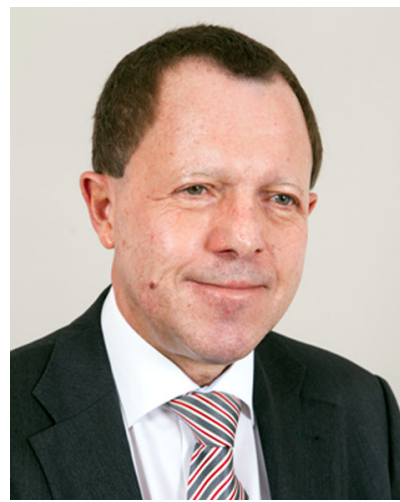

Prok. Dipl.-Ing. Johannes Vavra

\begin{abstract}
Sehr geehrte Damen und Herren,

ich begrüße Sie sehr herzlich zu unserer traditionellen Veranstaltung, welche heuer in Villach stattfindet, nämlich zur 54. Fachtagung der Österreichischen Gesellschaft für Energietechnik (OGE) im OVE.

Als Titel der diesjährigen OGE-Fachtagung haben wir die durchaus spannende Frage "Ist die Zukunft der Mobilität elektrisch?" gewählt. Aber warum wandte sich die Österreichische Ge-
\end{abstract}

sellschaft für Energietechnik diesmal eigentlich dem Thema Mobilität zu?

Nun, unter Berücksichtigung der vorjährigen OGE-Fachtagung, wo wir uns eingehend mit der Energiewende befassten, ist die Wahl des heurigen Themenschwerpunkts gewissermaßen als konsequente Fortsetzung zu betrachten, nimmt die Mobilität doch einen derart großen Anteil des Endenergiebedarfs ein, dass sie - ebenso wie etwa auch die Raumwärme - bei einer seriösen Beschäftigung mit der Energiewende selbstverständlich nicht vernachlässigt werden darf.

Ob die Zukunft der Mobilität darüber hinaus elektrisch sein wird, ist eine weiterführende Fragestellung, die sich beispielsweise aus den Beschlüssen der UN-Klimakonferenz in Paris 2015 (COP 21) ableiten lässt, muss doch ein Verzicht auf fossile Brennstoffe zwangsläufig zu einem solchen Ergebnis führen. Demgemäß wird sich die Fachtagung einer Vielzahl von themenspezifischen Gesichtspunkten widmen und vor allem auch eine Reihe von möglichen Antworten und Lösungsansätzen präsentieren.

Die inhaltliche Bandbreite erstreckt sich dabei von einer politischen Betrachtung der Bedeutung von Elektromobilität für Öster- reich, der Sichtweise der Regulierungsbehörde zur Elektromobilität, der technischen Rolle von Mobilität im zukünftigen Energiesystem bis zu Mobilitätskonzepten für Bahnen und urbane Räume, schließlich zu Fragen der Netz- und Systemintegration von Elektromobilität sowie Entwicklungen und Trends in der Ladeinfrastruktur und bei Fahrzeugbatterien.

Insgesamt gilt es, dem Anspruch einer Fachtagung zu genügen und daher auch konkrete Antworten in Form von Konzepten oder bereits realisierten organisatorischen und technischen Lösungen zu bieten. Hochkarätige Referenten aus Elektrizitätswirtschaft und Wissenschaft sowie Industrie, Behörden und Politik stehen folglich an diesen beiden Tagen zur Verfügung, um die Herausforderungen darzustellen und notwendige Maßnahmen zu beschreiben, Lösungsansätze vorzustellen, die Themen aus unterschiedlichen Blickwinkeln zu beleuchten und umfassend zu diskutieren.

Natürlich lade ich Sie, sehr geehrte Teilnehmerinnen und Teilnehmer, dazu ein, sich ebenfalls intensiv an diesen Diskussionen zu beteiligen und Ihre diesbezüglichen Erfahrungen einzubringen, darüber hinaus die Gelegenheit zum Netzwerken sowie auch zur Teilnahme an einer der beiden, am morgigen Nachmittag stattfindenden Exkursionen zu nutzen.

Abschließend möchte ich darauf hinweisen, dass im Rahmen der heutigen Abendveranstaltung in den Bambergsälen, zu der ich Sie natürlich gleichfalls herzlich einladen darf, wieder die Verleihung der OGE-Preise und des Prof. Werner Rieder-Preises erfolgen wird.

Ich wünsche allen Teilnehmerinnen und Teilnehmern sowie den Referentinnen und Referenten eine interessante, qualitativ hochwertige Veranstaltung und darf Sie somit auf der OGE-Fachtagung 2016 hier in Villach sehr herzlich willkommen heißen!

Dipl.-Ing. Johannes Vavra Vorstandsvorsitzender der Österreichischen Gesellschaft für Energietechnik (OGE) im OVE

Wiener Netze GmbH
Begrüßung bei der 54. Fachtagung der Österreichischen Gesellschaft für Energietechnik (OGE) im OVE, die am 24 und 25 November 2016 in Villach stattfand.

Vavra, Johannes, Wiener Netze GmbH, Erdbergstraße 236, 1110 Wien, Österreich (E-Mail: johannes.vavra@wienernetze.at) 